\title{
Aplicación de una técnica no convencional con efectos sobre el flujo de acceso: acupuntura
}

\author{
Gustavo Samuel Aguilar Gómez, Antonio José Fernández Jiménez, Irene Cabrera Plaza
}

\section{Centro de Hemodiálisis Sierra Este, Diálisis Andaluza. Sevilla}

\section{Introducción:}

La Acupuntura es una técnica milenaria que Enfermería aplica dentro de sus intervenciones, muchas veces sin conciliar las teorías tradicionales de las cuales procede. Diversos estudios ponen de manifiesto efectos vasculares a distintos niveles, con formas de aplicación diversas de la misma técnica, aunque sin conocer con exactitud sus mecanismos de actuación. Nos planteamos qué ocurriría en distintas constantes del paciente renal y en el flujo sanguíneo de su acceso, independientemente de su anastomosis, tras aplicar Acupuntura utilizando puntos a los que se les ha atribuido tradicionalmente efectos vasculares, pero a diferencia de estudios precedentes aplicando una estimulación distinta: Sedación.

\section{Objetivos:}

Detectar si existen diferencias en el flujo de acceso (QA) medido con Dilución Ultrasónica, en la Tensión Arterial (TAS, TAD, TAM) y en la Frecuencia Cardiaca (FC) tras la aplicación de Acupuntura Sistémica con Estímulo de Sedación.

\section{Material y método:}

Estudio cuasiexperimental Intrasujeto a $n=34$ pacientes en Hemodiálisis portadores de Acceso Vascular (AV) con trayecto lineal suficiente. Consentimiento informado. Dentro de una última sesión de hemodiálisis de la semana, realizamos las siguientes mediciones:

1. Mediciones iniciales dobles consecutivas de las variables en condiciones basales y determinación de sus medias aritméticas: MediaTASi, MediaTADi, TAMi, MediaFCi, MediaQAi.

2. Primera medición de las variables tras la punción de los puntos sin rotación de las agujas: TAS1, TAD1, TAM1, FCl y QA1.

3. Segunda medición de las variables tras un primer estímulo de sedación; rotando manualmente las agujas con amplitud $>180^{\circ}$, baja frecuencia y en contra del sentido de las agujas del reloj durante 6 minutos en los puntos 9 de Meridiano de Pulmón, 6 de Meridiano de Maestro Corazón y 36 de Meridiano de Estómago: TAS2, TAD2, TAM2, FC2 y QA2.

4. Tercera medición de las variables tras un segundo estímulo de sedación con las mismas características que en la fase anterior: TAS3, TAD3, TAM3, FC3 y QA3.

5. Cuarta medición de las variables seguidamente tras la retirada de las agujas Acupunturales: TAS4, TAD4, TAM4, FC4 y QA4.

El método que empleamos para las determinaciones del QA fue el descrito por Krivitski.

\section{Resultados:}

Características de la muestra: edad 65,97 \pm 13,13 años, 34 AVs: 1 prótesis Húmero-axilar (2,9\%) y 33 FAVIs $(97,1 \%)$. Estadísticos media \pm desviación estándar:

MediaTASi $124,69 \pm 21,75$, TAS1 $127,94 \pm 23,76$, TAS2 $129,41 \pm 25,22$, TAS3 $128,32 \pm 24,82$.

MediaTADi 64,46 $\pm 11,97$, TAD1 65,35 $\pm 11,85$, TAD2 $65,47 \pm 11,66$, TAD3 66,29 $\pm 13,31$. 
MediaFCi $74,31 \pm 10,86, \quad F C 1 \quad 76,09 \pm 11,71, \quad F C 2$ $73,21 \pm 10,89$, FC3 73,09 $\pm 10,39$.

MediaQAi $1234,85 \pm 614,781$, QAl $1346,47 \pm 737,32$, QA2 $1308,53 \pm 675,04$, QA3 $1275,88 \pm 628,73$, QA4 $1293,53 \pm 611,88$.

\section{Discusión y conclusiones:}

La sola aplicación de las agujas sin manipulación ha aumentado significativamente el QA de los AV; respuesta que ha sido mantenida con la primera estimulación propuesta. La insistencia con la segunda estimulación no provoca respuesta. También se produce un aumento significativo de la TAS tras la segunda estimulación y de la TAM tras la retirada de las agujas. La tendencia de la FC en cambio es a disminuir a lo largo de la intervención; siendo significativa tras la primera estimulación respecto a la aplicación de las agujas. No se presentaron incidencias sistémicas, ni sobre los puntos Acupunturales.

\section{Referencias Bibliográficas}

1. Varios autores. Guía de acceso vascular en hemodiálisis. SEN. 2004.

2. Nacional Kidney Foundation. K/DOQI Clinical Practice Guidelines for Vascular Access. Am J Kidney Dis 2001; 37 S137-S181.

3. Declaración de Beijing. Congreso de la OMS sobre Medicina Tradicional, Beijing (China), 8 de noviembre de 2008.

4. Directrices sobre capacitación básica y seguridad en la acupuntura. WHO/EDM/TRM/99.1.

5. Che-Yi C, Wen CY, Min-Tsung K, Chiu-Ching H. Acupuncture in haemodialysis patients at the Quchi
(LIII) acupoint for refractory uraemic pruritus. Nephrol Dial Transplant. 2005 Sep;20(9):1912-5. Epub 2005 Jun 28.

6. Sun $H$, Qiu MY, Li BQ, Wang SH, Chen $Z Y$, Jiang $Y$, Luan J. Effect of moxibustion on quality of life in hemodialysis patients. Zhongguo Zhen Jiu. 2008 May;28(5):321-4.

7. Stener V. E, Urban W., Sven A.A., Matts W. Reduction of blood flow impedance in the uterine arteries of infertile women with electro-acupuncture. Human Reproduction, Vol.11, No.6, 1996.

8. Aguilar GS, Fernández AJ, Cabrera I. Efectos Vasculares sobre las Fístulas Arteriovenosas Internas Radiocefálicas o de Cimino-Brescia de la Acupuntura Zonal con Estímulo de Tonificación (AZET). En: libro de comunicaciones del XXXV Congreso de la Sociedad Española de Enfermería Nefrológica; Granada 20-23 Octubre 2010. SEDEN; 2010.

9. Aguilar GS, Fernández AJ, Cabrera I. Influencia de la Acupuntura Zonal con Estímulo de Sedación (AZES) sobre la Tensión Arterial, Frecuencia Cardíaca y Flujo de Acceso de la FAVI Radiocefálica medido con Dilución Ultrasónica. En: libro de comunicaciones del XXXV Congreso de la Sociedad Española de Enfermería Nefrológica; Granada 2023 Octubre 2010. SEDEN; 2010.

10. Lian Y-L, Chen C-Y, Hammes M, Kolster B.C. Atlas Gráfico de Acupuntura Seirin. $1^{a}$ ed. : Könemann Verlagsgesellschaft mbH; 2000.

11. Krivitski NM. Theory and validation of access flow measurement by dilution technique during hemodialysis. Kidney International, Vol 481995 Jul: 244-250. 12. Krivitski N.M: Novel method to measure access flow during hemodialysis by ultrasound velocity dilution tecnique. ASOIO J41: M 741-M 745.1995. 